\title{
CLIC pre-alignment - status and remaining challenges
}

\author{
Juha Kemppinen ${ }^{1, *} \mathbb{D}$, Vivien Rude ${ }^{1}$, Hélène Mainaud Durand ${ }^{1}$ and Jouni Mattila ${ }^{2}$ (D) \\ ${ }^{1}$ CERN, Geneva, Switzerland \\ ${ }^{2}$ Tampere University, Tampere, Finland \\ E-mail: kemppi83@gmail.com
}

Received 6 November 2020, revised 30 April 2021

Accepted for publication 9 June 2021

Published 23 July 2021

\begin{abstract}
The Compact Linear Collider (CLIC) is a study of a $3 \mathrm{TeV}$ linear electron-positron $\left(\mathrm{e}^{+} \mathrm{e}^{-}\right)$ accelerator and is a successor candidate for CERN's Large Hadron Collider. The CLIC luminosity target is $5.9 \times 10^{34} \mathrm{~cm}^{-2} \mathrm{~s}^{-1}$, which causes unprecedented pre-alignment requirements of its main linear accelerator (main linac). Along the $50 \mathrm{~km}$ long tunnel, the main components of any $200 \mathrm{~m}$ long section have to be positioned within $10 \mu \mathrm{m}$ from a straight reference line. The pre-alignment challenge has been studied at CERN since the 1990s, and the main technical challenges have been solved. This article summarizes the positioning strategy and presents it to an audience outside the particle accelerator community. The methods can be of interest especially in the field of large-scale metrology. The positioning strategy consists of several steps or subsystems. The development of a straight reference line over tens of kilometers allows absolute positioning of accelerator components, while a process called fiducialization defines component reference axes with regard to alignment targets. Emphasis is on a support pre-alignment network that acts as a link between the straight reference line and fiducialization. The subsystems and remaining challenges in their development are presented. The chosen strategy's potential is demonstrated experimentally by building a short test setup.
\end{abstract}

Keywords: alignment, straight line reference, wire position sensor, stretched wire

(Some figures may appear in colour only in the online journal)

\section{Introduction}

CERN [1] is an international physics laboratory, situated on the border between France and Switzerland near the city of Geneva. The Compact Linear Collider (CLIC) study is an international collaboration led by CERN [2]. In its final form, CLIC will be a $50 \mathrm{~km}$ long linear electron-positron $\left(\mathrm{e}^{+} \mathrm{e}^{-}\right)$ collider. It is proposed as a successor candidate for the current flagship accelerator, the Large Hadron Collider (LHC). Hadron colliders like the LHC are optimized for exploring new energy levels, whereas $\mathrm{e}^{+} \mathrm{e}^{-}$accelerators produce cleaner

\footnotetext{
Author to whom any correspondence should be addressed.
}

Original Content from this work may be used under the terms of the Creative Commons Attribution 4.0 licence. Any further distribution of this work must maintain attribution to the author(s) and the title of the work, journal citation and DOI. collisions, making them better for studying the details of certain energy ranges. The CLIC conceptual design report [3], proving the overall feasibility of CLIC, was released in 2012. Since then, the study has been more detailed, concentrating on the implementation, cost and production issues of the different technologies involved.

CLIC is proposed to be built in three stages in order to distribute the costs over time. The building process of each stage takes several years, so it is also beneficial to be able to do physics research at lower energies between the building cycles. CLIC will provide $\mathrm{e}^{+} \mathrm{e}^{-}$collisions with center-of-mass energies of $380 \mathrm{GeV}, 1.5 \mathrm{TeV}$ and $3 \mathrm{TeV}$ with configurations that are housed in 12.1, 29.6 and $50.7 \mathrm{~km}$ long main tunnels respectively [4]. Figure 1 shows the scale of the stages on a map [5].

An update to the European Strategy for Particle Physics was published in June 2020 [6]. It declares that a technical and financial feasibility study for a next-generation hadron collider 


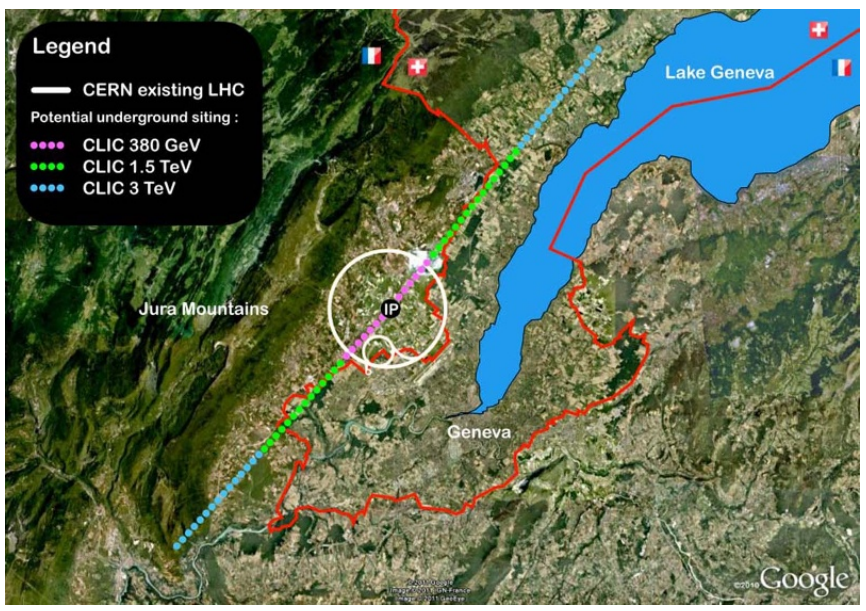

Figure 1. The three stages of CLIC at its potential location near CERN [5]. Reproduced from [5]. CC BY 4.0.

is a priority in preparation for the long-term. This means that the 1.5 and $3 \mathrm{TeV}$ CLIC options are no longer considered. However, the strategy update recommends a so-called Higgs factory as the highest priority to follow the LHC. The $380 \mathrm{GeV}$ CLIC is a strong candidate for this. This article puts emphasis on the $3 \mathrm{TeV}$ CLIC. Some of the tolerances presented below could be relaxed by a factor of two if it is certain that only the $380 \mathrm{GeV}$ CLIC is built [7]. Otherwise, the technical requirements, especially on pre-alignment and positioning, are also valid for the $380 \mathrm{GeV}$ CLIC.

Figure 2 shows the CLIC $3 \mathrm{TeV}$ layout overview. This article concentrates on the $\mathrm{e}^{+}$and $\mathrm{e}^{-}$main linear accelerators (linacs), which accelerate the $\mathrm{e}^{+}$and $\mathrm{e}^{-}$beams respectively and deliver them to the beam delivery systems (BDSs), which do the final preparations for collisions at the interaction point (IP).

CLIC is based on a two-beam acceleration scheme in which the radio frequency (RF) power that is used to accelerate the particle beam is extracted from a low-energy but high-intensity drive beam (DB). The acceleration itself is done by RF accelerating structures (ASs), which are situated on the main beam (MB). The power is extracted from the DB by power extraction and transfer units and transferred to the MB by waveguides. Both beams also have quadrupole magnets, called MBQ and $\mathrm{DBQ}$, which focus the beams. Each MBQ is equipped with a beam position monitor (BPM), which measures the transversal position of the beam passing through the MBQ.

The $380 \mathrm{GeV}$ CLIC could also be built without DB. The RF power would in this case be generated by RF amplifiers called klystrons. This is a more traditional approach in particle accelerators. Regardless of the powering option, the MB and its associated pre-alignment requirements (presented below) are the same $[4,5]$.

\subsection{Luminosity}

In a particle accelerator like CLIC, two beams of particles travel close to the speed of light in opposite directions and collide in the middle. An important parameter of an accelerator is the luminosity $\mathcal{L}$, which means the number of collisions per area per unit of time. The smaller the transverse size of the beam $\sigma$, the higher the luminosity. Furthermore, the beam size is proportional to the beam emittance $\varepsilon$, which is a measure of the average particle coordinate spread. In the $3 \mathrm{TeV}$ CLIC, the target $\mathcal{L}=5.9 \times 10^{34} \mathrm{~cm}^{-2} \mathrm{~s}^{-1}$ [5]. This causes a high charge density, which leads to strong beam-beam effects, resulting in emission of radiation called beamstrahlung [8] and production of background particles. These harmful parameters are kept acceptable by using flat beams, which are significantly larger in the horizontal than in the vertical direction [9]. $\sigma_{x}=40 \mathrm{~nm}$, $\sigma_{y}=1 \mathrm{~nm}, \varepsilon_{x}=660 \mathrm{~nm}$ and $\varepsilon_{y}=20 \mathrm{~nm} \mathrm{[3].}$

One of the key challenges of CLIC is the preservation of low emittance when the beams travel through the accelerator complex. If the beam trajectory is not straight, beam particles with different energies will take slightly different paths, leading to an increase in the beam emittance. The trajectory is monitored by the BPMs, so their misalignment with respect to the beam has the highest impact on emittance growth, followed by ASs misalignment [7]. Beam-based alignment corrects these static imperfections, but it can only work as foreseen if all the main linac components are mechanically prealigned accurately [3].

\subsection{Pre-alignment}

The pre-alignment error budget is defined as shown in figure 3 . The reference axes of all individual components of a certain type have to be located, in an absolute reference frame, within a certain radius from a straight reference line along sliding windows of $200 \mathrm{~m}$ (any $200 \mathrm{~m}$ long section of the main linac). The radius is $14 \mu \mathrm{m}$ for the electrical zeros of BPMs and the electro-magnetic axes of the ASs, $17 \mu \mathrm{m}$ for the magnetic axes of the MBQs and $20 \mu \mathrm{m}$ for the magnetic axes of the DBQs. The positioning requirement is even more demanding $-10 \mu \mathrm{m}$ —in the BDS, which consists of the last $2.75 \mathrm{~km}$ on both sides of the IP (see figure 2) [4].

The pre-alignment requirements that stem from the CLIC luminosity target are unprecedented in particle accelerators. For example, in the LHC, the fiducials (see section 2.3) of dipole magnets and other components are mechanically aligned to within $0.1 \mathrm{~mm}$. During technical stops, with intervals of several months, traditional surveying instruments are used to verify the alignment. The accelerator components are installed on manually adjustable jacks, which are used to position the components according to the survey.

An important part of alignment, especially for a linear accelerator, is the creation of a straight reference line. SLAC has been a pioneer in the field. The final focus test beam had strict requirements over $300 \mathrm{~m}$-absolute alignment of $100 \mu \mathrm{m}$ in radial and $30 \mu \mathrm{m}$ in vertical. After the initial alignment, on-line monitoring was done with a relative alignment tolerance of $15 \mu \mathrm{m}$ in radial and $5 \mu \mathrm{m}$ in vertical [10].

There are many x-ray free-electron laser (XFEL) projects around the world. SwissFEL at the Paul Scherrer Institute (PSI) in Switzerland is aligned within $50 \mu \mathrm{m}$ in short range and relative [11]. The European XFEL, coordinated by DESY in Germany, has an alignment goal of $0.3 \mathrm{~mm}$ over $150 \mathrm{~m}$ and 


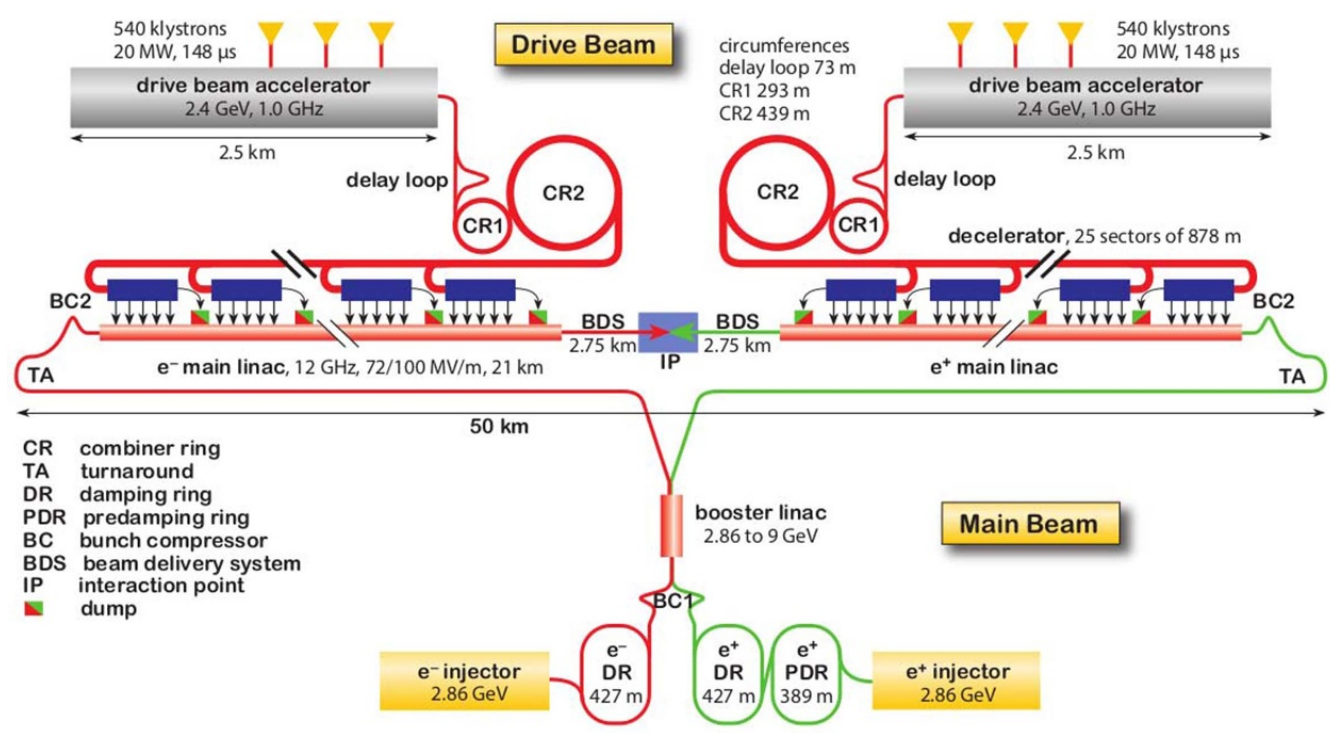

Figure 2. Overview of the layout of the CLIC $3 \mathrm{TeV}$ configuration [5]. Reproduced from [5]. CC BY 4.0.

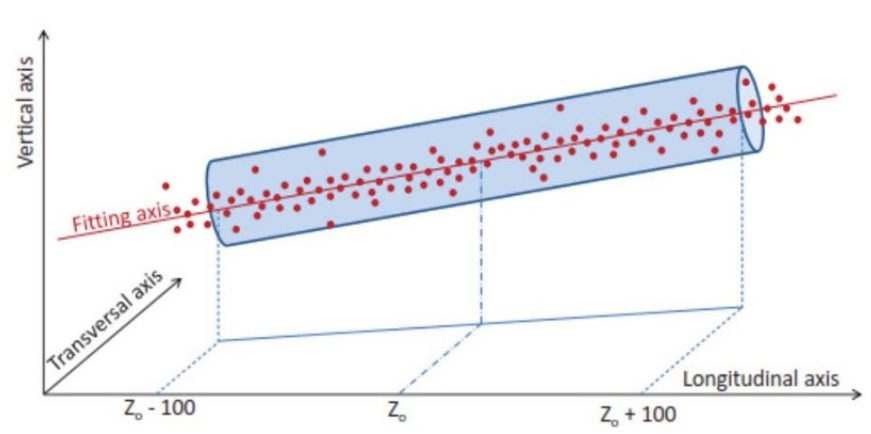

Figure 3. CLIC pre-alignment requirement. All reference axes of a certain component type have to be located within a defined radius (e.g. BPM electrical zeros $14 \mu \mathrm{m}$ ) from a straight reference line on any $200 \mathrm{~m}$ window [3]. Reproduced from [3]. CC BY 3.0.

for the connection between monochromators and undulators $0.5 \mathrm{~mm}$ over $1000 \mathrm{~m} \mathrm{[12].}$

Modern synchrotron light sources have their components aligned in tens of micrometers relative to each other. For example, $60 \mu \mathrm{m}$ for the Extremely Brilliant Source at the European Synchrotron Radiation Facility (ESRF) in France [13] and $25 \mu \mathrm{m}$ for the SPring-8-II in Japan [14]. A good summary of the alignment requirements for particle accelerators can be found in [15].

The above mentioned projects do not have the same requirement level as CLIC pre-alignment. This is especially true since CLIC alignment tolerances are set in absolute terms in the $200 \mathrm{~m}$ sliding windows.

When making the leap from the $0.1 \mathrm{~mm}$ level to the $10 \mu \mathrm{m}$ level, the whole positioning strategy changes. Seismic ground movements, cultural noise, human and industrial activity, as well as temperature variations, become so significant that components need to be frequently readjusted [16]. They remain aligned for hours or days instead of months or years. Stopping CLIC daily for personnel access is not feasible. Instead, alignment has to be monitored continuously and corrected when needed, remotely and automatically.

In order to limit the number of items to align, many of the main components are mounted on $2 \mathrm{~m}$ long common supports called girders before they are installed in the tunnel. Even so, there will be 40000 items to monitor and position. It is thus clear that cost is an important design parameter.

CLIC pre-alignment has been studied at CERN since the 1990s. The main technical challenges have been solved, and many articles and conference papers have been published on the topic. This article summarizes the positioning strategy and presents it to an audience outside the particle accelerator community. The methods can be of interest, especially in the field of large-scale metrology.

The positioning strategy consists of several steps. To enable an absolute frame of reference, the CERN coordinate system (CCS; already defined) is brought to the tunnel. Through an intermediary step, a straight reference line for the length of the whole main linac is created based on stretched wires. Component reference axes are defined in a local coordinate system (CS) by a measurement process called fiducialization. The local CSs are accurately transformed to the general reference (materialized by stretched wires) by a support pre-alignment network (SPN). The main components are positioned with respect to the SPN by positioning systems.

Wire position sensors (WPSs) and their calibration process are introduced in section 2.1. Sections 2.2-2.4 present the above-mentioned steps of positioning strategy. Sections 3 and 4 are dedicated to the main topic of the article - SPN. Section 3 presents formulas to calculate the position of a component in the general CS. This has not been published before. The effectiveness of the chosen strategy is demonstrated in section 4, which presents a short test setup that was built at CERN, and the results obtained from it. 


\section{Positioning strategy}

The CLIC pre-alignment error budget has been defined in an absolute reference frame. This means that a well-defined and precise CLIC CS needs to be defined. The CCS needs to be combined with a geodetic reference frame. In addition, a geodetic model, built based on astro-zenithal, GPS and gravimetric measurements, is needed. CLIC will have shafts linking the tunnel to the surface every $2.5 \mathrm{~km}$. Geodetic pillars will be installed near the shafts on the surface, and they will be determined in the surface model. The model is then transferred from the pillars to the tunnel via the shafts with an uncertainty of measurement of $\pm 1 \mathrm{~mm}$ using a combination of mechanical and optical measurements. Between the shafts in the tunnel, an underground geodetic network that consists of points in the floor every $50 \mathrm{~m}$ is determined with relative uncertainty of $\pm 0.1 \mathrm{~mm}[17,18]$.

\subsection{Wire position sensor}

Before presenting the next step in the positioning strategy, an important instrument needs to be introduced. The WPS, shown in figure 4(a), is a capacitive sensor manufactured by Fogale Nanotech. It measures the vertical and lateral offsets of a stretched wire that goes through it. Measurement range is $10 \mathrm{~mm} \times 10 \mathrm{~mm}$ and precision in relative measurements $1 \mu \mathrm{m}$ [19].

The WPS is used to measure the positions of bodies it is installed on. It is mounted on the body through Kelvin kinematic couplings, shown in figure 4(b). Three ceramic spheres of $8 \mathrm{~mm}$ diameter and ISO 3290 grade $40(1 \mu \mathrm{m}$ spherical form tolerance) are glued to the metrological plate. The WPS has an interface with a conical cup, a V-groove and a flat surface. Even though the conical cup is in line contact with the ceramic sphere and the coupling is thus over constrained, the repeatability of the coupling is below $1 \mu \mathrm{m}$. The coordinate vectors $\overrightarrow{u_{x}}$, $\overrightarrow{u_{y}}$ and $\overrightarrow{u_{z}}$ of figure 4(b) can be calculated based on the sphere coordinates. To complete the coupling CS, its origin is placed at the WPS theoretical zero [17].

The CLIC positioning strategy is based on a WPS that is accurately calibrated in absolute. The kinematic coupling plays a crucial role in the process. It links the sensor voltages to the CS where the ceramic spheres are defined. Two-phased calibrations are needed. In the first phase, the WPS is calibrated relative to a linearity bench. Afterward, the sensor readings are linked to the coupling in an absolute calibration bench.

A linearity calibration bench for the WPS was developed at CERN. The bench has a lateral and a vertical displacement table stacked (XY-guide). Both have a displacement resolution of $10 \mathrm{~nm}$ over a distance of $75 \mathrm{~mm}$. A kinematic coupling for the WPS is installed on the XY-guide. When a WPS is being calibrated, it is mounted on the coupling, and a wire is stretched through it. The XY-guide displacements needed to be verified when the bench was built. An interferometer would have been the best instrument for this in terms of resolution and precision. However, it is precise in one direction, whereas it is important for us to measure the sphere positions in 3D. In this way, the misalignment between the XY-guide stages is

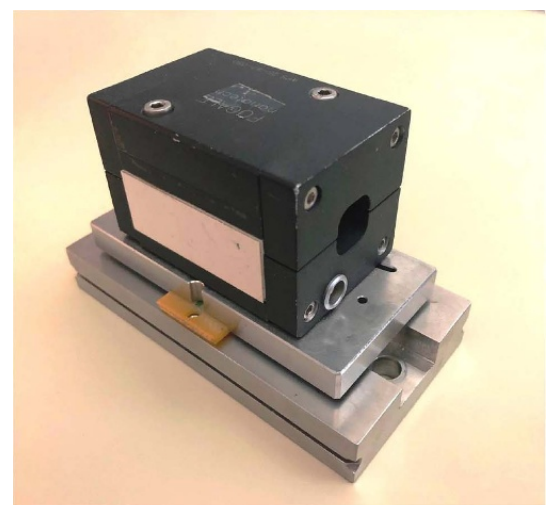

(a)

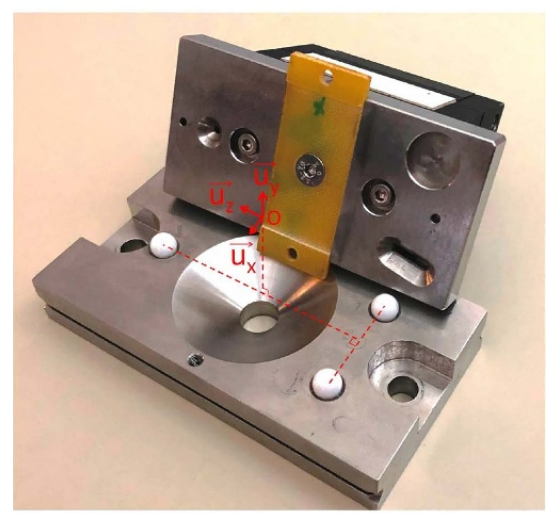

(b)

Figure 4. (a) Capacitive wire position sensor (WPS). (b) Kinematic coupling positions the WPS accurately on the metrological plate.

taken into account in the calibration. Therefore, a Leitz Infinity coordinate measuring machine (CMM) with a maximum permissible error (MPE) for length measurement (MPEE) of $0.3 \mu \mathrm{m}+1 \mathrm{ppm}$ was chosen. In the same measurement process, a coordinate system was created for the linearity bench.

The lateral and vertical WPS electrodes have 0-10 V voltage outputs. The outputs are $5 \mathrm{~V}$ when the wire goes through the sensor center. After the WPS is mounted on the bench and the wire is stretched through it, the XY-guide is used to search the position where the WPS output is $5 \mathrm{~V}$. After this, 127 pre-defined displacements are made. From this data, a polynomial of degree 36 is fitted for both offsets. The polynomials transform $\mathrm{V}$ to $\mathrm{mm}$.

It has been shown that the WPS positions along $\overrightarrow{u_{z}}$, as well as around $\overrightarrow{u_{x}}$ and $\overrightarrow{u_{y}}$, need to be defined within $1 \mathrm{~mm}$ and $1^{\circ}$, respectively [20]. These, as well as the rotation around $\overrightarrow{u_{z}}$ (within $500 \mu \mathrm{rad}$ ), are extracted directly from CMM measurements of the kinematic coupling. The remaining two degrees of freedom (DOF) - translations along $\overrightarrow{u_{x}}$ and $\overrightarrow{u_{y}}$ - need to be calibrated accurately [17].

The linearity table gives the relative calibration. An absolute calibration bench was also developed at CERN, and is presented in figure 5. The principle is illustrated on the right. The calibration bench has a CS R0. There are four kinematic couplings, R1-R4, which have been defined accurately in R0 using the Leitz Infinity CMM. The photo on the left shows 

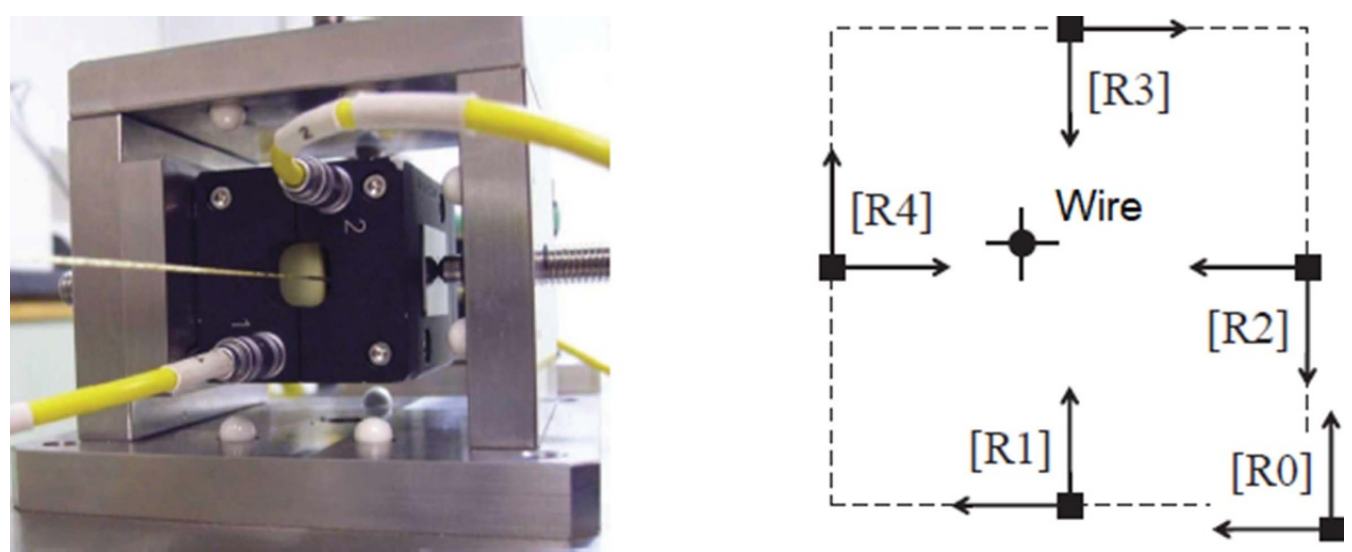

Figure 5. WPS absolute calibration bench, which links the sensor centre to the ceramic spheres of the kinematic coupling (R-sensor WPS) [17]. Reproduced with permission from [17].

how the kinematic couplings have materialized. After the wire position has been measured by the WPS mounted on one kinematic coupling, the WPS is moved to another coupling without touching the wire in between. By comparing the wire position measurements from the four different couplings, the sensor readings in mm can be linked to the CS of the ceramic spheres (R-sensor WPS) [17, 19]. The combined absolute calibration has an accuracy better than $5 \mu \mathrm{m}$. This value also takes into account the uncertainty of the shape of the wire $[19,21]$. The WPS location is changed by hand, which is a delicate operation. Calibrating one WPS takes a long time. In CLIC, there would be tens of thousands of sensors. This means that the process would have to be automated.

The wire could be made of any material that conducts electricity. Other properties, like how much tension can be applied to the wire, and what its mass is, finally decide the suitable wire for each case. For example, Carbon PEEK and Carbon KEVLAR have been used in CLIC studies. WPS calibration is valid only when the WPS uses wire from the same batch as the wire used in the calibration.

\subsection{Metrological reference network}

This article follows a convention where the coordinate axes of the CLIC CS R-general are defined in the following way. $Z$ is the direction of the beam, $Y$ is the vertical (positive pointing upward) and $X$ is the vector fulfilling a cartesian CS. $A, B$ and $C$ are rotations around $X, Y$ and $Z$, respectively.

Ruland has proposed to classify alignment systems based on their main feature - the straight line reference (SLR). The classification is between an optical and a mechanical straight line, as well as gravity [22]. The CLIC metrological reference network (MRN) creates straight reference lines along the whole main linac. A combination of a mechanical straight line (stretched wires) and a gravity reference (hydrostatic network) was chosen.

Stretched wires are used because they can be mathematically modeled by a straight line in the horizontal plane and a catenary curve in the vertical plane. It is not possible to stretch a single wire over the whole main linac length. Therefore,

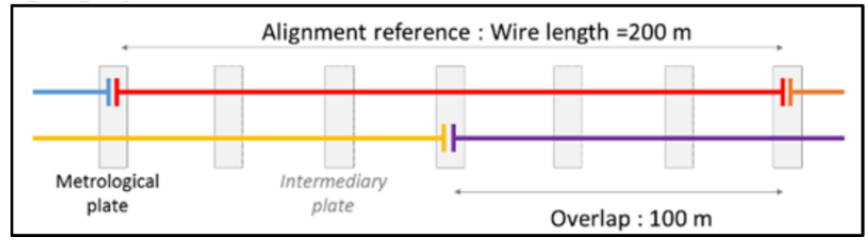

Figure 6. Metrological reference network schematic [21]. Reproduced from [21]. CC BY 4.0.

the MRN consists of two series of parallel wires that overlap. Wires should be as long as possible to minimize error propagation. However, long wires take up a lot of space because the sag (see section 3.2) grows with length. Taking both aspects into account, the wire length choice in CLIC was $200 \mathrm{~m}$ and the wires overlap by $100 \mathrm{~m}$. Figure 6 illustrates the wire configuration [21]. Wire overlapping limits the propagation of error.

A metrological plate, shown in figure 7 , is located at each wire extremity. The coordinates of the ceramic spheres of the three WPS supports are measured a priori in a CMM in the referential frame of the metrological plates (R-plate). A 3D transformation from R-sensor WPS to R-plate can be deduced from the $a$ priori data (the transformation equations of section 3.1 can be used). The MRN also contains intermediary plates (see figure 6), which add redundancy [21].

The stretched wires will be defined in absolute and thus all WPS measurements are also in absolute. Therefore, the wires do not have to be parallel. The only limiting factor is the size of the WPS opening $(10 \mathrm{~mm} \times 10 \mathrm{~mm})$.

In order to have an accurate model of the wire catenary, the vertical offset between at least three points of the wire should be known. In order to improve this precision, a hydrostatic network is added to the MRN. Hydrostatic leveling sensors (HLSs), which measure the vertical offset with respect to a hydraulic network, are installed on all metrological and intermediary plates of figure 6 [21].

It would be very beneficial to have a hydrostatic network over the whole length of CLIC. In principle, there would be no error propagation, and MRN could be defined very accurately in the vertical direction. The problem is that the water surface 


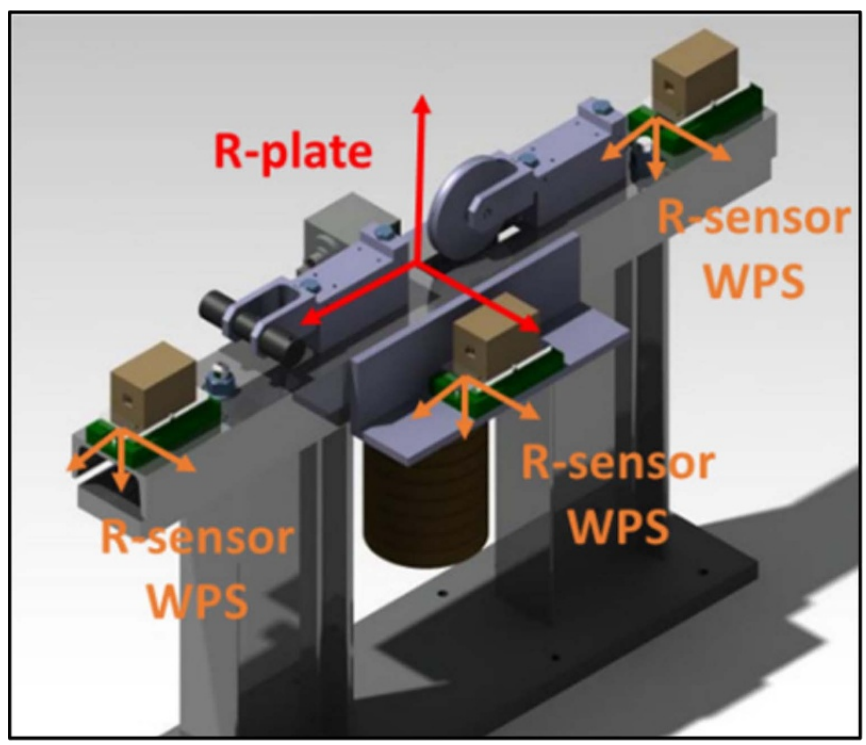

Figure 7. Metrological plate of the metrological reference network [21]. Reproduced from [21]. CC BY 4.0.

along the network follows gravity equipotential, which is not known accurately. Defining it has been studied for CLIC, but additional studies would be needed [23].

The MRN has been confirmed to reach CLIC requirements in a $140 \mathrm{~m}$ long test setup, and it correlates well with simulations [21]. Before building CLIC, a $200 \mathrm{~m}$ long test setup would be needed in order to define final parameters - for example, the number and distribution of intermediary plates (see figure 6). In addition to the MRN presented above, an optical SLR based on lasers has been studied. This project, called LAMBDA, got promising results in a $35 \mathrm{~m}$ long test setup. A $200 \mathrm{~m}$ long LAMBDA test setup should be built together with an MRN one in order to make an intercomparison. This would not only decide which system to use in CLIC, but would also give reliable data on both systems' performance [25].

\subsection{Fiducialization}

Fiducialization is the measurement process where a component's reference axis is linked to external metrological targets, called fiducials. In CLIC, the reference axes are the electrical zeros of the BPMs, electro-magnetic axes of the ASs, and magnetic axes of the MBQs and DBQs. Ceramic spheres that are attached to the outside of the component are used as fiducials. Fiducialization is important because the reference axes cannot usually be accessed after the accelerator has been installed, while the alignment should be done with respect to the reference axis [26].

In a project called PACMAN, $10 \mathrm{PhD}$ students developed methods to accurately fiducialize the most important CLIC main linac component types. The reference axes of all CLIC main linac component types can be determined by stretching a wire through the beam line of the component. Figure 8 shows the Final PACMAN Alignment Bench (FPAB), where a wire made of copper and beryllium $(\mathrm{Cu} 98 \%, \mathrm{Be} 2 \%)$ is stretched

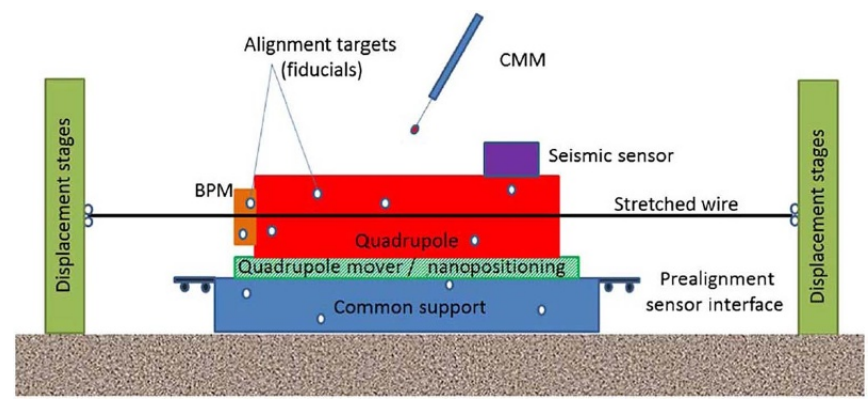

Figure 8. Schematic of the final PACMAN alignment bench (FPAB) [24]. Reproduced from [24]. CC BY 4.0.

between precise displacement stages. The wire goes through a combination of an MBQ and a BPM. The displacement stages move the wire until the MBQ and BPM reference axes are found.

PACMAN studied the reference axis definition of all component types mentioned at the beginning of this section. To study the AS, the conducting wire is used to perturb the first dipole mode excited in the AS at $17 \mathrm{GHz}$ using a network analyzer. The electro-magnetic zero is searched by moving the wire to a position where the perturbation is minimized [27]. For BPM, the wire position has an impact on the electromagnetic field of the excited dipole mode of the cavity. This interference of the dipole eigen-mode takes its minimum when the wire is positioned on the BPM electrical zero axis [28]. The vibrating wire method was applied and further developed to determine the MBQ magnetic axes [29].

FPAB is installed on a CMM, as shown in figure 8. The wire is measured using a touchless probe and the fiducials using a traditional probe. A new touchless probe that takes into account the wire's form error was developed [30]. All measurement data are directly in the same CS, which limits uncertainty. Accurate mobile measurement devices based on micro triangulation [31] and frequency scanning interferometry [32] were also developed so that fiducialization can be done outside the laboratory [33].

In order to reach the pre-alignment requirements presented in figure 1.2, the fiducialization uncertainty has to be below $12 \mu \mathrm{m}$. It was shown that the technologies developed during the PACMAN project can reach this goal [34].

MBQs and their associated BPMs will be positioned separately in CLIC. Each MBQ is equipped with four WPS supports (see figure 4(b)). Other types of components are installed on common $2 \mathrm{~m}$ long girders. During the PACMAN project, a method to position the components accurately on girders during fiducialization was developed [18]. All girders are also equipped with four WPS supports. The fiducialization process defines the WPS positions of both MBQs and girders in their CSs (R-girder).

\subsection{Positioning systems}

The MRN (section 2.2) provides the straight reference line. Fiducialization (section 2.3) gives component reference axes and alignment sensors in R-girder. Two more systems are 
needed to complete CLIC active pre-alignment. The SPN links fiducialization to the MRN and defines the necessary transformations from the component reference axis to R-general. The SPN is the article's main topic, and it is presented in section 3. It is not enough to know the girder and MBQ positions; they also need to be adjusted. They are thus equipped with motorized positioning systems with micrometer redsolution in five DOF (translation along $Z$ is not as important and can be mechanically blocked).

It has been demonstrated that linear actuators can be used to position the girders in the DB side [35]. All DB girders are linked to one another, and each joint is equipped with two vertical and a horizontal linear actuator. This snake-like configuration allows positioning of the girders in five DOF within requirements.

The main beam is more challenging. Because of the mix between girders and MBQs, the snake-like configuration is not practical. It has been shown that a parallel kinematic configuration of five eccentric cam shafts, called a cam mover, meets the CLIC positioning precision requirements in 5 DOF [36]. However, blocking translation in $Z$ while maintaining the positioning precision has not yet been demonstrated. For this reason, and because of a new requirement to position the MB girders also in $Z$-translation, a six-axis cam mover has been proposed [18]. The MBQ positioning system has an additional stiffness requirement [37], which has not yet been met simultaneously with the micrometer resolution requirement.

\section{Support pre-alignment network}

As mentioned in section 2.3, CLIC MB AS and all DB components will be installed on $2 \mathrm{~m}$ long common girders. They are fiducialized and adjusted iteratively so that they are well aligned in the girder CS (R-girder). Contrary to other types of components, MBQs are not installed on girders. Instead, their positions will be adjusted and measured directly. Both the girders and MBQs are equipped with four WPSsw each. Thanks to the calibration and fiducialization processes that were presented in section 2, the WPSs measure component reference axis locations in girder or MBQ CS (R-girder).

The SPN acts as the link between fiducialization and the MRN. From the SPN point of view, girders and MBQs can be considered to be equivalent. As stated in section 2.4, girders and MBQs will be equipped with either a five DOF positioning system where translation along the beam is mechanically blocked or with a six DOF positioning system. The girder and MBQ target positions will be calculated by a smoothing algorithm that requires their current positions as inputs.

Figure 9 shows the configuration of stretched wires for both the MB and the DB. The two inner wires form the MRN (metrological plates, see figure 6) and part of the SPN. With only the inner wires, MBQ and girder position parameters $X$, $Y, A$ and $B$ can be measured in R-general. Adding the outer wires and thus the number of WPSs per girder and MBQ from two to four enables also accurate $C$ parameter measurement. It also brings redundancy (eight measurement points for five unknown parameters). Approximate positions along the

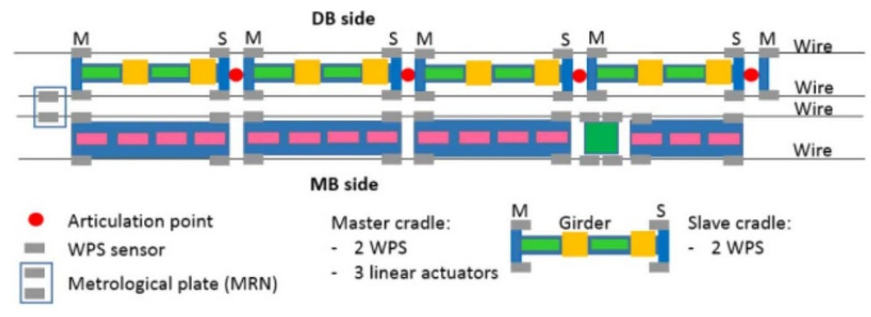

Figure 9. Main beam and drive beam sensor and girder configuration [18]. Reproduced with permission from [18].

beam $Z$ have to be measured during installation with other methods.

The length of the wire as well as the design and distribution of metrological and intermediary plates of the outer wires have not yet been determined. The very first metrological plate of CLIC will be fixed and house a WPS for all four stretched wires. After that, the wires do not have to be perfectly parallel. In fact, it is enough that all wires pass through all their designated WPS. Positions of all other metrological and intermediary plates can be adjusted during their installation and, if needed, afterward during a maintenance break. The four stretched wires will be installed and defined before the girders and MBQs are installed. This means that when the WPSs are installed around the stretched wires, their positions in R-general will be known.

In the short test setup, which is presented in section 4, there are two metrological plates and one MBQ between them. Two wires are stretched between the metrological plates, one on each side of the MBQ. The goal is to simulate the SPN by creating a similar measurement situation to any of the girders or MBQ in figure 9.

The metrological plates of the test setup are considered to be fixed. Metrological targets are attached to them. The targets are measured in local (R-plate) and general (R-general) CS. In section 3.1, the formulas of transformation through geometric similarity are presented.

Sections 3.1-3.3 contain least-squares adjustment and error propagation through the adjustment. These methods are frequently used in topography, and the equations are derived e.g. in [38].

\subsection{Transformation to R-general}

In this section, the transformation from R-girder or R-plate to $\mathrm{R}$-general is defined. The transformation parameters are collected in vector $\boldsymbol{x}$, which is calculated using the least squares:

$$
\boldsymbol{x}=\left[\begin{array}{lllllll}
X & Y & Z & A & B & C & \tau
\end{array}\right]^{T},
$$

where $X, Y$ and $Z$ are translation parameters and $A, B$ and $C$ are rotations around the $X, Y$ and $Z$-axes, respectively. $\tau$ is the scale factor. The observation equation of the transformation is:

$$
g(\boldsymbol{x})=M_{j}=\left[\begin{array}{lll}
X & Y & Z
\end{array}\right]^{T}+\tau \boldsymbol{R}_{z} \boldsymbol{R}_{y} \boldsymbol{R}_{x} m_{j}
$$

where $m_{j}$ is a target point $j$ in R-plate and $M_{j}$ is the same point in R-general. The rotation matrices around $X, Y$ and $Z$ ( $s$ means sine and $c$ means cosine) are: 


$$
\begin{aligned}
& \boldsymbol{R}_{x}=\left[\begin{array}{ccc}
1 & 0 & 0 \\
0 & c A & -s A \\
0 & s A & c A
\end{array}\right], \\
& \boldsymbol{R}_{y}=\left[\begin{array}{ccc}
c B & 0 & s B \\
0 & 1 & 0 \\
-s B & 0 & c B
\end{array}\right], \\
& \boldsymbol{R}_{z}=\left[\begin{array}{ccc}
c C & -s C & 0 \\
s C & c C & 0 \\
0 & 0 & 1
\end{array}\right] .
\end{aligned}
$$

In every iteration, the $m$ items of the $m$-by-1 parameter vector $\boldsymbol{x}$ are updated:

$$
\boldsymbol{x}^{(k+1)}=\boldsymbol{x}^{(k)}+\hat{\boldsymbol{x}}
$$

The convergence criterion is that the L 2 norm of the leastsquares result vector $\hat{\boldsymbol{x}}$ is below $\varepsilon$ (equal to $1 \mathrm{~nm}$ in the study). The least-squares solution is:

$$
\hat{\boldsymbol{x}}=\boldsymbol{N}^{-1} \boldsymbol{C}=\left(\boldsymbol{A}^{T} \boldsymbol{P} \boldsymbol{A}\right)^{-1} \boldsymbol{A}^{T} \boldsymbol{P} \boldsymbol{b},
$$

where $\boldsymbol{N}^{-1} \boldsymbol{C}$ is called a system of normal equations, in which $\boldsymbol{N}=\boldsymbol{A}^{T} \boldsymbol{P A}$ is rectangular, symmetric, and generally positive definite and reversible. The least-squares condition is:

$$
\boldsymbol{A}^{T} \boldsymbol{P} \boldsymbol{v}=0
$$

$\boldsymbol{A}$ is the n-by-m Jacobian matrix of $g\left(\boldsymbol{x}^{(k)}\right), n$ is the number of observations, which is three times the number of target spheres ( $X, Y$ and $Z$ coordinates), and $m$ is the number of parameters to fit (length of $\boldsymbol{x}$ ):

$$
\boldsymbol{A}_{i,:}=\left[\begin{array}{ccc}
\frac{\partial g_{1}\left(\boldsymbol{x}^{k}\right)}{\partial \boldsymbol{x}_{1}^{k}} & \cdots & \frac{\partial g_{1}\left(\boldsymbol{x}^{k}\right)}{\partial \boldsymbol{x}_{m}^{k}} \\
\vdots & \ddots & \vdots \\
\frac{\partial g_{n}\left(\boldsymbol{x}^{k}\right)}{\partial \boldsymbol{x}_{1}^{k}} & \cdots & \frac{\partial g_{n}\left(\boldsymbol{x}^{k}\right)}{\partial \boldsymbol{x}_{m}^{k}}
\end{array}\right]
$$

$\boldsymbol{P}$ is an $n$-by- $n$ weight matrix:

$$
\boldsymbol{P}=\left[\begin{array}{ccc}
1 / \sigma_{1}^{2} & \cdots & 0 \\
\vdots & \ddots & \vdots \\
0 & \cdots & 1 / \sigma_{n}^{2}
\end{array}\right]
$$

where $\sigma_{i}$ is the a priori uncertainty of observation $i . \sigma_{i}$ is calculated on every iteration through a Monte Carlo simulation, which is based on a coordinate transformation using the current iteration $\boldsymbol{x}^{(k)}$ and the measurement uncertainties of the CMM and the laser tracker:

$$
\boldsymbol{v}=\boldsymbol{A x}-\boldsymbol{b}
$$

which contains the deviations between the model and the observations. $\boldsymbol{b}$ is called the measurement vector:

$$
\boldsymbol{b}=\boldsymbol{l}-\boldsymbol{g},
$$

where $\boldsymbol{l}$ is the observation vector, containing the laser tracker measurements $M_{j}$. The vector $g$ contains the metrology measurements $m_{j}$ that have been transformed using equation (2) with the current iteration $\boldsymbol{x}^{(k)}$ and the observation that corresponds to the row:

$$
\boldsymbol{g}_{i}=g_{i}\left(\boldsymbol{x}^{k}\right)
$$

\subsection{Stretched wire model}

Through the absolute calibration of the sensors, WPS voltages can be converted to measurement points in the plate and component CSs. The metrological plates (and intermediary plates) are considered to be stationary, and the transformations from their local coordinates to the general ones are defined above. The other components (girders and MBQs), on the other hand, can be positioned using positioning systems so their positions need to be defined and monitored continuously after the installation using the WPS.

$T_{x}$ and $T_{y}$ are the $X$ - and $Y$-translations of a component and unknown. $T_{Z}$ is less important, and it cannot be measured using the WPS, so the $Z$ coordinate is taken directly from the laser tracker measurement data and considered known. $\theta_{x}, \theta_{y}$ and $\theta_{z}$ are the component rotations around the coordinate axes and also unknown. The rotation matrix $\boldsymbol{R}=\boldsymbol{R}_{z} \boldsymbol{R}_{y} \boldsymbol{R}_{x}$ is calculated using equations (3)-(5), replacing $A, B$ and $C$ with $\theta_{x}, \theta_{y}$ and $\theta_{\mathrm{z}}$, respectively.

The stretched wires in figure 9 link the metrological plates to the components that can be positioned. Since the WPSs measure points that are constrained by the wires, the mobile components' positions can be calculated by modeling the wires and knowing the Z-positions of all WPS along the wires. Since the transformations of the plates are known, the remaining unknown parameters are the $X$ - and $Y$-coordinates of the first WPS of each wire, and the offsets of the $X$ - and $Y$ coordinates between the first and the last WPS of each wire, $\Delta X$ and $\Delta Y$. The $m=13$ unknown parameters are gathered in $\boldsymbol{x}$ :

$$
\boldsymbol{x}=\left[\begin{array}{c}
X_{1} \\
\Delta X_{1} \\
Y_{1} \\
\Delta Y_{1} \\
X_{2} \\
\Delta X_{2} \\
Y_{2} \\
\Delta Y_{2} \\
T_{x} \\
T_{y} \\
\theta_{x} \\
\theta_{y} \\
\theta_{z}
\end{array}\right] .
$$

The generalized least-squares method, presented through equations (2)-(13), can be used again to solve $\boldsymbol{x}$. The $n$-by-1 vector $l$ contains the $n=16$ WPS observations (eight sensors measuring $X$ - and $Y$-offsets), transformed to R-general. The remaining task is to find the observation equation $g(\boldsymbol{x})$. 
The wires can be modeled as straight lines in the lateral and catenary curves in the vertical direction. To model the catenary, the wire sag is calculated [39]:

$$
f=\frac{g q l^{2}}{8 T}
$$

where $g$ is the gravitational acceleration, $q$ is the linear mass of the wire, $l$ is the longitudinal distance of the wire ends, and $T$ is the tension applied to the wire. As mentioned in the MRN section, the wire sag model accuracy can be improved by adding a hydrostatic network. In the next section, the approach is validated using a short test setup. The wire catenaries can be modeled accurately without HLSs. Thus, for simplicity, the hydrostatic network is omitted in this article. Its modeling principle can be found in [17]. The next step is to calculate the wire sag at each WPS location:

$$
f_{i}=\frac{4 f \Delta Z_{j}^{2}}{l^{2}}+\frac{h-4 f \Delta Z_{j}}{l}
$$

where $Z_{j}$ is the distance between WPS $j$ and the beginning of the wire along $Z$, and $h$ is the vertical offset between the two ends of the wire.

The vector $\boldsymbol{g}$ has 16 items, like $\boldsymbol{l}$. The observation equation $g(\boldsymbol{x})$ is not the same for each item of $\boldsymbol{g}$ this time. Each WPS produces two items, based on the $X$ and $Y$-measurements respectively. If the WPS is located on a metrological plate:

$$
\boldsymbol{g}(\boldsymbol{x})=\left[\begin{array}{c}
X_{i} \\
Y_{i}
\end{array}\right]+\frac{\Delta Z_{j}}{l}\left[\begin{array}{c}
\Delta X_{i} \\
\Delta Y_{i}
\end{array}\right]+\left[\begin{array}{l}
0 \\
f_{i}
\end{array}\right] .
$$

Since the metrological plates are at the extremities, $f_{i}=0$. If the WPS is located on a component, $g(\boldsymbol{x})$ includes transformation from the local CS to R-general:

$$
\boldsymbol{g}(\boldsymbol{x})=\boldsymbol{R}^{T}\left(\left[\begin{array}{c}
X_{i} \\
Y_{i} \\
Z_{i}
\end{array}\right]+\frac{\Delta Z_{j}}{l}\left[\begin{array}{c}
\Delta X_{i} \\
\Delta Y_{i} \\
0
\end{array}\right]+\left[\begin{array}{c}
0 \\
f_{i} \\
0
\end{array}\right]-\left[\begin{array}{c}
T_{x} \\
T_{y} \\
T_{z}
\end{array}\right]\right)
$$

Both $Z_{i}$ and $T_{z}$ are taken from the laser tracker measurement data and since they are known, only the two first lines of $\boldsymbol{g}(\boldsymbol{x})$, corresponding to $X$ - and $Y$-directions, are included in the vector $g$.

The weight matrix $\boldsymbol{P}$ is calculated using equation (10) and $\sigma_{i}$ is the a priori uncertainty of observation $i$. All observations of $\boldsymbol{l}$ are made with the WPS sensors. They are considered equally precise with each other and in both directions. $\sigma_{i}$ is the precision of the absolute calibration process, and it includes the uncertainties of the CMM and the laser tracker.

\subsection{Measurement accuracy}

The next step is to calculate how the observational uncertainties propagate to the parameters $\boldsymbol{x}$ that were calculated using the least-squares method. The following formulas apply to both the coordinate transformation and the wire model. In the following, it is assumed that the observations are independent (diagonal covariance matrix) and that the random errors have centered normal distributions. The $n$-by- $n$ a priori covariance matrix of observations $\boldsymbol{\Sigma}_{L}$ is the inverse of the weight matrix $\boldsymbol{P}$ (see equation (10)). The real covariance matrix of observations is then:

$$
\boldsymbol{\Sigma}_{L}^{\prime}=s_{0}^{2} \boldsymbol{\Sigma}_{L}
$$

where $s_{0}^{2}$ (in $\mathbb{R}$ ) is called the variance factor of the unit weight. One can estimate the exactness of the a priori uncertainties from $s_{0}^{2}$. If it is exactly 1 , the a priori uncertainties are correct. Below 1 means that they are pessimistic and above 1 means that they are optimistic. The exact value of $s_{0}^{2}$ cannot be known, but it can be estimated:

$$
\hat{\boldsymbol{s}}_{0}^{2}=\frac{\hat{\boldsymbol{v}}^{T} \boldsymbol{\Sigma}_{L}^{-1} \hat{\boldsymbol{v}}}{n-m}=\frac{\hat{\boldsymbol{v}}^{T} \boldsymbol{P} \hat{\boldsymbol{v}}}{n-m},
$$

where

$$
\hat{\boldsymbol{v}}=\boldsymbol{A} \hat{\boldsymbol{x}}-\boldsymbol{b}
$$

is the $n$-by- 1 vector of measurement residuals. $\hat{\boldsymbol{x}}$ and $\boldsymbol{b}$ are taken from the last iteration, when the least squares converge. The assumption that the random errors have centered normal distributions leads to an assumption that $\hat{\boldsymbol{v}}$ follows a normal distribution. Then, $\hat{\boldsymbol{v}}^{T} \boldsymbol{P} \hat{\boldsymbol{v}}$ should have $\chi^{2}$ distribution of $n-m$ DOF. The goodness of the fit can be evaluated by testing whether this is the case. A null hypothesis $\mathcal{H}_{0}$ that $s_{0}^{2}=1$ is made. $(n-m) \hat{s}_{0}^{2}$ should be close to 1 and found in the interval $\left[\gamma_{1}, \gamma_{2}\right]$ with a chosen probability $\alpha$. The border values $\gamma_{1}$ and $\gamma_{2}$ can be found in inverse $\chi^{2}$ table with probability levels $(1-\alpha) / 2$ and $(1+\alpha) / 2$, respectively, as well as $(n-m)$ DOF. Depending on whether the $\chi^{2}$ test succeeded or not, the $n$-by- $n$ covariance matrix of the residuals is calculated using equation (22) or (23) correspondingly:

$$
\begin{gathered}
\boldsymbol{\Sigma}_{\hat{\boldsymbol{v}}}=\hat{s}_{0}^{2}\left(\boldsymbol{P}^{-1}-\boldsymbol{A} \boldsymbol{N}^{-1} \boldsymbol{A}^{T}\right) \\
\boldsymbol{\Sigma}_{\hat{\boldsymbol{v}}}=\boldsymbol{P}^{-1}-\boldsymbol{A} \boldsymbol{N}^{-1} \boldsymbol{A}^{T} .
\end{gathered}
$$

The diagonal variance terms of $\boldsymbol{\Sigma}_{\hat{v}}$, denoted as $\hat{\zeta}_{i}$, are used to normalize the residuals:

$$
\hat{\boldsymbol{w}}_{i}=\frac{\hat{\boldsymbol{v}}_{i}}{\sqrt{\hat{\zeta}_{i}}}
$$

The normalized residuals can be validated by knowing that they follow a Student's $\tau$ distribution with $n-m$ DOF. A posteriori variance-covariance matrices of the fitted parameters $\boldsymbol{x}$, depending on whether the $\chi^{2}$ test succeeded (equation (25)) or not (equation (26)):

$$
\begin{aligned}
& \Sigma_{x}=\hat{s}_{0}^{2} N^{-1} \\
& \Sigma_{x}=N^{-1} .
\end{aligned}
$$

Fitted parameter precisions are the square roots of the diagonal terms:

$$
\boldsymbol{\omega}_{i}=\sqrt{\boldsymbol{\Sigma}_{\boldsymbol{x}_{i, i}}} .
$$




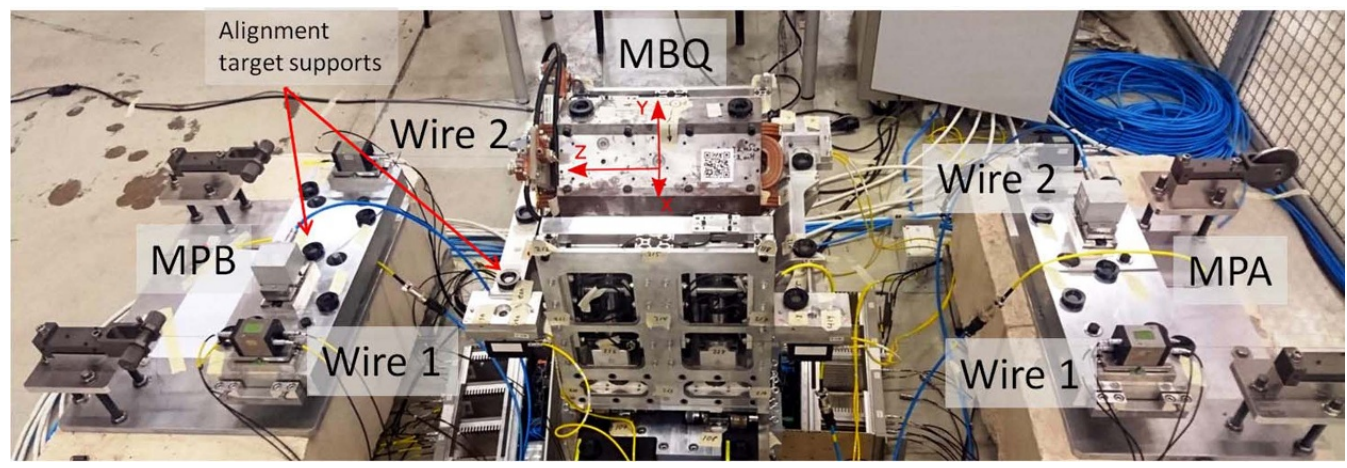

Figure 10. Test setup containing a prototype MBQ and the alignment sensors to measure its position accurately.

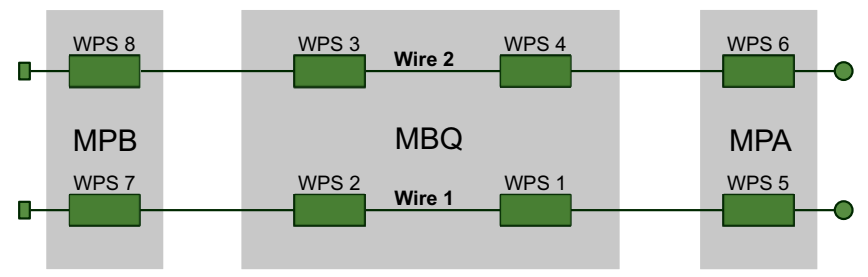

Figure 11. CLIC main beam quadrupole pre-alignment test setup schematic. The setup consists of two metrological plates (MPA and MPB), an MBQ, two stretched wires and eight WPS sensors.

\section{Experimental setup}

A short experimental setup, shown in figure 10, was built at CERN. It simulates the SPN that links the girder or MBQ fiducialization to the MRN. The MBQ position in figure 10 is measured just like any girder or MBQ position in figure 9. The goal was to demonstrate the CLIC positioning strategy's accuracy in short distance.

Figure 11 shows the same test setup configuration. It consists of an MBQ and two metrological plates, $\mathrm{A}$ and $\mathrm{B}$ (MPA and MPB hereafter). Two stretched wires and a total of eight WPS sensors are installed in the setup. With such short stretched wires, the catenaries can be modeled accurately without a hydrostatic network. For convenience, the R-general origin is located at the center of the MBQ on the beam line, and it is not attached to the MBQ.

\subsection{Measurements before installation}

Before the test setup was installed, the ceramic spheres of all WPS supports were measured in the local CS (R-girder and R-plate). In order to perform the transformations presented in section 3.1, additional magnetic metrology target supports were attached to both plates. MBQ also has additional targets for measurement of its location along $Z$. Some of the target supports are visible in figure $10 ; 1.5$ inch prisms or spheres can be attached to the supports during measurements.

The plates MPA and MPB have five target supports each, and MBQ has eight. The measurement of one of the points on plate B was unsuccessful (statistical test), and it had to be dropped. In addition, MPA and MPB have two WPS each, and
MBQ has four. WPS supports consist of three $8 \mathrm{~mm}$ ceramic spheres; see section 2.1 .

The MBQ points were measured using a Prismo Ultra CMM before installation. Its MPE is $0.0012 \mathrm{~mm}+\mathrm{L} / 500 \mathrm{~mm}$. The MPA and MPB points were measured a priori with a Leica AT401 laser tracker. Its MPE is $0.75^{\prime \prime}$ in angular and $0.008 \mathrm{~mm} \pm 1 \mathrm{ppm}$ in distance. $\sigma_{i}$ of the MBQ and plate point observations are then estimated to be 0.002 and $0.010 \mathrm{~mm}$, respectively. These numbers stem from previous experience with the instruments, and were verified after measurements with statistical tests (see section 3.3).

The results are gathered in table 1 . It contains only the 1.5 inch target measurements because they are used to determine the transformation from R-plate to R-general. The WPS coordinates after transformation to R-general are published in tables 3 and 4. The targets on the MBQ are used in the next section to measure the MBQ position in $Z$.

\subsection{Measurements after installation}

After installation of the setup, the 1.5 inch targets were measured in a common CS using the Leica AT401 laser tracker. The laser tracker was measuring the targets from three different positions, called stations, during the same afternoon. The results were linked using a built-in feature called unified spatial metrology network (USMN) [40] of the software SpatialAnalyzer $^{\circledR}$ (SA) by Hexagon Metrology. The $a$ posteriori precisions of the measurement were $0.86^{\prime \prime}$ (horizontal angle), $0.86^{\prime \prime}$ (vertical angle) and distance $0.007 \mathrm{~mm}$. The results are gathered in table 2 .

\subsection{Transformations and analysis}

Tables 1 and 2 list the positions of the same targets but in different CS. They and formulas of section 3.1 were used to define transformations from MPA and MPB local CS to Rgeneral as well as MBQ position in $Z$.

The a priori and laser tracker measurements were used to calculate transformations using geometric similarity. These transformations were used to calculate the WPS positions in R-general. The maximum wire sag was then calculated with equation (15), where $g=9.81 \mathrm{~m} \mathrm{~s}^{-2}, q=2.35 \times 10^{-4} \mathrm{~kg} \mathrm{~m}^{-1}$ (linear mass of the wire Carbon Peek), $l=1.453 \mathrm{~m}$ and 
Table 1. Plate and MBQ target points in their local reference frames, measured with Prismo Ultra CMM (MBQ) and Leica AT401 laser tracker.

\begin{tabular}{|c|c|c|c|c|c|c|}
\hline & $x(\mathrm{~mm})$ & $y(\mathrm{~mm})$ & $z(\mathrm{~mm})$ & $\boldsymbol{\sigma}_{x}(\mathrm{~mm})$ & $\boldsymbol{\sigma}_{y}(\mathrm{~mm})$ & $\boldsymbol{\sigma}_{z}(\mathrm{~mm})$ \\
\hline MBQ1 & -154.213 & -73.005 & 258.434 & 0.002 & 0.002 & 0.002 \\
\hline MBQ2 & 9.068 & -72.221 & 257.546 & 0.002 & 0.002 & 0.002 \\
\hline MBQ3 & 180.18 & -72.222 & 257.506 & 0.002 & 0.002 & 0.002 \\
\hline MBQ4 & -157.942 & -85.119 & -249.976 & 0.002 & 0.002 & 0.002 \\
\hline MBQ5 & 89.138 & -84.807 & -244.83 & 0.002 & 0.002 & 0.002 \\
\hline MBQ6 & 216.26 & -80.38 & -224.976 & 0.002 & 0.002 & 0.002 \\
\hline MBQ7 & 0.694 & 143.516 & 128.313 & 0.002 & 0.002 & 0.002 \\
\hline MBQ8 & 4.803 & 142.861 & -131.764 & 0.002 & 0.002 & 0.002 \\
\hline MPA1 & -210.752 & 2.624 & -132.513 & 0.010 & 0.010 & 0.010 \\
\hline MPA2 & -119.416 & 2.607 & 16.273 & 0.010 & 0.010 & 0.010 \\
\hline MPA3 & 32.437 & 2.697 & -63.035 & 0.010 & 0.010 & 0.010 \\
\hline MPA4 & 103.139 & 2.624 & 13.282 & 0.010 & 0.010 & 0.010 \\
\hline MPA5 & 215.408 & 2.604 & -132.081 & 0.010 & 0.010 & 0.010 \\
\hline MPB1 & -209.934 & 2.685 & 61.92 & 0.010 & 0.010 & 0.010 \\
\hline MPB2 & -121.525 & 2.671 & -88.618 & 0.010 & 0.010 & 0.010 \\
\hline MPB3 & -17.607 & 2.644 & -14.662 & 0.010 & 0.010 & 0.010 \\
\hline MPB4 & 122.679 & 2.687 & -90.706 & 0.010 & 0.010 & 0.010 \\
\hline MPB5 & 210.26 & 2.673 & 60.067 & 0.010 & 0.010 & 0.010 \\
\hline
\end{tabular}

Table 2. Plate and MBQ target points in R-general, measured with a laser tracker.

\begin{tabular}{|c|c|c|c|c|c|c|}
\hline & $x(\mathrm{~mm})$ & $y(\mathrm{~mm})$ & $z(\mathrm{~mm})$ & $\sigma_{x}(\mathrm{~mm})$ & $\sigma_{y}(\mathrm{~mm})$ & $\sigma_{z}(\mathrm{~mm})$ \\
\hline MBQ1 & -154.584 & -73.099 & 257.955 & 0.005 & 0.005 & 0.004 \\
\hline MBQ2 & 8.72 & -71.815 & 257.469 & 0.009 & 0.008 & 0.008 \\
\hline MBQ3 & 179.838 & -71.293 & 257.867 & 0.008 & 0.008 & 0.008 \\
\hline MBQ4 & -157.026 & -83.316 & -250.529 & 0.007 & 0.006 & 0.005 \\
\hline MBQ5 & 90.064 & -82.275 & -244.777 & 0.007 & 0.006 & 0.005 \\
\hline MBQ6 & 217.133 & -77.541 & -224.589 & 0.009 & 0.008 & 0.009 \\
\hline MBQ7 & -0.006 & 144.379 & 129.008 & 0.005 & 0.005 & 0.004 \\
\hline MBQ8 & 4.749 & 144.719 & -131.067 & 0.006 & 0.005 & 0.004 \\
\hline MPA1 & -230.921 & -207.214 & -859.056 & 0.011 & 0.012 & 0.011 \\
\hline MPA2 & -139.399 & -207.328 & -710.365 & 0.006 & 0.005 & 0.005 \\
\hline MPA3 & 12.368 & -207.23 & -789.829 & 0.007 & 0.006 & 0.006 \\
\hline MPA4 & 83.152 & -207.345 & -713.617 & 0.006 & 0.005 & 0.005 \\
\hline MPA5 & 195.281 & -207.307 & -859.111 & 0.008 & 0.006 & 0.006 \\
\hline MPB1 & -230.001 & -207.281 & 788.553 & 0.009 & 0.008 & 0.008 \\
\hline MPB2 & -141.489 & -207.528 & 638.085 & 0.006 & 0.006 & 0.005 \\
\hline MPB3 & -37.614 & -207.443 & 712.129 & 0.007 & 0.006 & 0.006 \\
\hline MPB4 & 102.756 & -207.494 & 636.132 & 0.005 & 0.005 & 0.005 \\
\hline MPB5 & 190.249 & -207.278 & 786.964 & 0.008 & 0.007 & 0.007 \\
\hline
\end{tabular}

Table 3. Wire 1 parameters.

\begin{tabular}{llllll}
\hline & $x(\mathrm{~mm})$ & $y(\mathrm{~mm})$ & $z(\mathrm{~mm})$ & $\Delta Z_{j}(\mathrm{~mm})$ & $f_{i}(\mathrm{~mm})$ \\
\hline WPS 5 & 244.220 & -173.373 & -762.056 & 0 & 0 \\
WPS 1 & 244.023 & -172.566 & -249.633 & 512.423 & -0.0036 \\
WPS 2 & 243.604 & -171.715 & 258.603 & 1020.661 & -0.0033 \\
WPS 7 & 243.455 & -171.097 & 691.924 & 1453.982 & 0 \\
\hline
\end{tabular}

$T=150 \mathrm{~N}$. This yields a sag of $4 \mu \mathrm{m}$. The next step was to calculate the wire sag at each WPS, $f_{i}$, using equation (16). In this setup, the wire ends are at the same heights $(h=0)$. The WPS coordinates, $\Delta Z_{j}$ and $f_{i}$ of wires 1 and 2 (see figure 11), are gathered in tables 3 and 4 respectively.
In the least-squares calculation of MBQ position, the uncertainties of the CMM and the laser tracker are added to the precision of the WPS absolute calibration process. The combined a priori $\sigma_{i}=0.005 \mathrm{~mm}$ for the WPS mounted on the MBQ and $\sigma_{i}=0.010 \mathrm{~mm}$ for the WPS mounted on the metrological 
Table 4. Wire 2 parameters.

\begin{tabular}{llllll}
\hline & $x(\mathrm{~mm})$ & $y(\mathrm{~mm})$ & $z(\mathrm{~mm})$ & \multicolumn{1}{c}{$\Delta Z_{j}(\mathrm{~mm})$} & $f_{i}(\mathrm{~mm})$ \\
\hline WPS 6 & -283.410 & -181.247 & -761.447 & 0 & 0 \\
WPS 4 & -282.884 & -180.797 & -252.239 & 509.208 & -0.0036 \\
WPS 3 & -282.557 & -180.192 & 257.441 & 1018.888 & -0.0033 \\
WPS 8 & -282.112 & -179.722 & 691.596 & 1453.044 & 0 \\
\hline
\end{tabular}

Table 5. MBQ position in R-general.

\begin{tabular}{lll}
\hline & Value & Precision $(1 \sigma)$ \\
\hline$T_{x}$ & $0.082 \mathrm{~mm}$ & $0.007 \mathrm{~mm}$ \\
$T_{y}$ & $1.342 \mathrm{~mm}$ & $0.006 \mathrm{~mm}$ \\
$\theta_{x}$ & $794.9^{\prime \prime}$ & $2.5^{\prime \prime}$ \\
$\theta_{y}$ & $-453.2^{\prime \prime}$ & $2.5^{\prime \prime}$ \\
$\theta_{z}$ & $595.1^{\prime \prime}$ & $4.3^{\prime \prime}$ \\
\hline
\end{tabular}

plates. The MBQ position and associated precisions are listed in table 5 .

There are 13 unknown parameters and 16 WPS observations, resulting in three DOF. With a 0.95 confidence level, $\hat{s}_{0}^{2}$ should be in the interval $[0.07,3.12]$. In this case $\hat{s}_{0}^{2}=1.85$, which means that the $\chi^{2}$ test is passed. However, the amount of DOF is low. Therefore, the precisions in table 5 are calculated using equation (26). The maximum normalized WPS residual is $0.005 \mathrm{~mm}$ on the MBQ and $0.009 \mathrm{~mm}$ on the metrological plates.

The test setup shows that at short distances, the positioning strategy can reach CLIC accuracy requirements. However, this measurement did not take into account the uncertainty of fiducialization. On the other hand, the metrological plates were not measured in a CMM, and the MBQ points were not measured with the most accurate CMM available at CERN. MBQ position can be monitored continuously with approximately the precisions indicated in table 5, except the direction along $Z$, which is less important.

\section{Conclusions}

The CLIC luminosity target provides stringent requirements for the pre-alignment of the main linac components. The reference axes of the main components of any $200 \mathrm{~m}$ long section have to be within a radius of $14-20 \mu \mathrm{m}$ from a straight reference line. This article presents the strategy that was adopted to meet the requirements.

An accurate reference line can be created based on stretched wires and capacitive WPSs. With the results of the PACMAN project, the components can be fiducialized and positioned on their common support girders very accurately at the same time as the WPS positions are measured in girder CS. Emphasis of the article was on SPN, which links MRN and fiducialization. The equations for calculating the girder (or MB quadrupole, which is positioned independently) positions with respect to the stretched wires were published. The remaining challenges were mentioned in the presentations of different systems in sections 2 and 3.
A short setup consisting of MBQ, two metrological plates, two stretched wires and a total of eight WPSs was used as an example. The MBQ position and orientation in five DOF were calculated based on a priori data and WPS measurements. The setup demonstrated that a component's position can be monitored according to CLIC pre-alignment requirements.

\section{Data availability statement}

The data that support the findings of this study are available upon reasonable request from the authors.

\section{ORCID iDs}

Juha Kemppinen (D) https://orcid.org/0000-0002-9714-2958

Jouni Mattila (D) https://orcid.org/0000-0003-1799-4323

\section{References}

[1] Cern website (available at: https://home.cern/)

[2] Clic study website (available at: http://clic-study.web.cern.ch/)

[3] Aicheler M et al 2012 A Multi-TeV Linear Collider Based on CLIC Technology: CLIC Conceptual Design Report (CERN Yellow Reports: Monographs) (Geneva: CERN) (https://doi. org/10.5170/CERN-2012-007)

[4] Aicheler M, Burrows P N, Catalan Lasheras N, Corsini R, Draper M, Osborne J, Schulte D, Stapnes S and Stuart M J 2018 The compact linear collider (CLIC) - project implementation plan CERN Yellow Rep.: Monogr. 4 46, 95, 149

[5] Sicking E 2016 Updated baseline for a staged compact linear collider CERN Yellow Rep. 4 27-37

[6] European strategy for particle physics (available at: https:// europeanstrategy.cern/european-strategy-for-particlephysics)

[7] Blaskovic Kraljevic N and Schulte D 2019 Beam-based beamline element alignment for the main linac of the 380 $\mathrm{GeV}$ stage of CLIC 10th Int. Particle Accelerator Conf. (IPAC'19) (Melbourne, Australia, 19-24 May 2019) pp 465-67

[8] Schroeder D V 1990 Beamstrahlung and QED backgrounds at future linear colliders Other Thesis

[9] Robson A, Burrows P N, Lasheras N C, Linssen L, Petric M, Schulte D, Sicking E, Stapnes S and Wuensch W 2018 The compact linear $\mathrm{e}^{+} \mathrm{e}^{-}$collider (clic): accelerator and detector (arXiv:1812.07987)

[10] Ruland R and Fischer G 1990 The proposed alignment system for the final focus test beam at slac 2nd Int. Workshop on Accelerator Alignment (Germany: Deutsches Elektronen-Synchrotron (DESY))

[11] Dreyer K and Höwler T 2012 Survey and alignment for the new Swissfel accelerator 12th Int. Workshop on Accelerator Alignment (IWAA 2012) (USA) 
[12] Kamtner D and Prenting J 2006 Straight line reference system (SLRS) for the adjustment of the $\mathrm{x}$-ray free-electron laser (XFEL) at DESY eConf. C06092511 TH008

[13] Martin D 2017 Alignment strategy for the new ESRF storage ring 3rd PACMAN workshop (available at: https:// indico.cern.ch/event/600191/contributions/2477575/)

[14] Zhang C, Azumi N, Fukami K, Kimura H, Kiuchi J, Matsui S, Takemura Y, Takano S and Watanabe T 2021 Magnet alignment monitoring system with eigenfrequency-based wire sag correction Meas. Sci. Technol. 32075009

[15] Leão R J, Baldo C R, da Costa Reis M L C and Trabanco J L A 2018 Engineering survey planning for the alignment of a particle accelerator: part I. Proposition of an assessment method Meas. Sci. Technol. 29034006

[16] Becker F, Coosemans W, Pittin R and Wilson I 2003 An Active Pre-Alignment System and Metrology Network for CLIC CLIC-NOTE-553, CERN-CLIC-NOTE-553, CERN-OPEN-2003-011 CERN

[17] Touzé T 2011 Proposition d'une méthode d'alignement de l'accélérateur linéaire CLIC PhD Thesis Paris-Est U

[18] Mainaud Durand H, Jaros J, Kemppinen J, Sosin M, Rude V and Zemanek A 2018 Clic pre-alignment strategy: final proposal and associated results 15th Int. Workshop on Accelerator Alignment

[19] Mainaud Durand H, Bestmann P, Herty A, Marin A and Rude V 2012 oWPS versus cWPS Technical Report CERN-ATS-2012-271 CERN Geneva

[20] Becker F 2003 Définition d'un réseau de référence métrologique pour le positionnement d'un grand accélérateur linéaire Theses INSA de Strasbourg

[21] Mainaud Durand H, Pfingstner J and Rude V 2017 Micrometric propagation of error using overlapping stretched wires for the CLIC pre-alignment 8th Int. Particle Conf. p TUIK098

[22] Ruland R E 1995 Some alignment considerations for the next linear collider eConf. C951114 045

[23] Guillaume S 2015 Determination of a precise gravity field for the CLIC feasibility studies PhD Thesis ETH Zurich Zürich

[24] Caiazza D, Catalan Lasheras N, Mainaud Durand H, Modena M, Sanz C, Tshilumba D, Vlachakis V, Wendt M and Zorzetti S 2017 New solution for the high accuracy alignment of accelerator components Phys. Rev. Accel. Beams 20083501

[25] Stern G 2016 Study and development of a laser based alignment system for the compact linear collider $\mathrm{PhD}$ Thesis ETH Zurich Zürich

[26] Griffet S, Cherif A, Kemppinen J, Mainaud Durand H, Rude V and Sterbini G 2012 Strategy and validation of fiducialisation for the pre-alignment of CLIC components Conf. Proc. C1205201 WED073

[27] Galindo Munoz N, Boria V, Catalán Lasheras N and Faus-Golfe A 2017 Pre-alignment techniques developments and measurement results of the electromagnetic center of warm high-gradient accelerating structures 8 th Int. Particle Conf.

[28] Zorzetti S, Wendt M and Fanucci L 2017 Study of the electrical center of a resonant cavity beam position monitor (RF-BPM) and its integration with the main beam quadrupole for alignment purposes 2 nd North American Particle Conf.

[29] Arpaia P, Caiazza D, Petrone C and Russenschuck S 2015 Performance of the stretched- and vibrating-wire techniques and correction of background fields in locating quadrupole magnetic axes Technical Report CERN, Geneva

[30] Sanz C, Giusca C, Morantz P, Marin A, Chérif A, Schneider J, Mainaud-Durand H, Shore P and Steffens N 2018 Form measurement of a $0.1 \mathrm{~mm}$ diameter wire with a chromatic confocal sensor, with associated uncertainty evaluation Meas. Sci. Technol. 29074010

[31] Vlachakis V, Fuchs J F and Mainaud Durand H 2016 Recent development of micro-triangulation for magnet fiducialisation 14th Int. Workshop on Accelerator Alignment

[32] Kamugasa S W, Gayde J C and Mainaud-Durand H 2016 Frequency scanning interferometry for CLIC component fiducialisation 14th Int. Workshop Accelerator Alignment

[33] Mainaud Durand Het al 2017 Main achievements of the pacman project for the alignment at micrometric scale of accelerator components 8th Int. Particle Conf. p TUIK077

[34] Doytchinov I P, Tonnellier X, Shore P, Nicquevert B, Modena M and Mainaud Durand H 2018 Application of probabilistic modelling for the uncertainty evaluation of alignment measurements of large accelerator magnets assemblies Meas. Sci. Technol. 29054001

[35] Sosin M 2016 Issues and feasibility demonstration of CLIC supporting system chain active pre-alignment using a multi-module test setup (mock-up) CERN-ACC-NOTE-2016-0063 CERN

[36] Kemppinen J, Mainaud Durand H and Kostka Z 2017 Cam mover alignment system positioning with wire position sensor feedback for clic Proc., 9th Mechanical Engineering Design of Synchrotron Radiation Equipment and Instrumentation (MEDSI 2016): (Barcelona, Spain, 11-16 September 2016) (Geneva, CERN) p MOE15

[37] Janssens S 2015 Stabilisation and precision pointing quadrupole magnets in the compact linear collider (CLIC) presented 14 Jan 2015 (available at: https://cds.cern.ch/ record/1985255)

[38] Wolf P R and Ghilani C 1995 Survey Measurement Adjustments by Least Squares (Boston, MA: Springer US) pp 383-413

[39] Mainaud H 1996 Une nouvelle approche métrologique: l'écartométrie biaxiale: application à l'alignement des accélérateurs linéaires $\mathrm{PhD}$ Thesis Université Louis Pasteur, Strasbourg

[40] Calkins J M 2002 Quantifying Coordinate Uncertainty Fields in Coupled Spatial Measurement systems PhD Thesis Virginia Polytechnic Institute and State University 